\title{
Variabilité génétique, héritabilités et corrélations de 15 caractères d'une population de clones de topinambour (Helianthus tuberosus L)
}

\author{
F Le Cochec
}

INRA, Station d'amélioration des plantes, Domaine de la Motte au Vicomte, BP 29, 35650 Le Rheu, France

(Reçu le 19 octobre 1989; accepté le 9 octobre 1990)

\begin{abstract}
Résumé - Quinze caractères en relation avec les stades de développement de la plante et le rendement ont été observés sur 16 clones de topinambour représentatifs de la variabilité existant dans une collection de clones. Pour tous les caractères, les différences entre clones sont très significatives et indiquent l'existence d'une variabilité importante. Les taux d'héritabilité au sens large sont compris entre 19,5 et $96,1 \%$. Ils sont très bas pour les caractères liés au rendement et très élevés pour le pourcentage de matière sèche des tubercules et les caractères en relation avec le développement de la plante. Les gains attendus en sélection, exprimés en proportion (\%) de la moyenne, varient de $9,5 \%$ pour la durée de la levée à $30,7 \%$ pour la hauteur des tiges. L'analyse des path-coefficients de 5 caractères a mis en évidence un effet direct et indirect important du poids sec de la plante entière sur le rendement en matière sèche des tubercules. Les valeurs moyennes des clones ont été analysées suivant la méthode des composantes principales en utilisant 4 axes de référence qui représentent $90 \%$ de la variabilité totale. La dispersion des clones le long du premier axe principal montre l'existence de 2 groupes morphologiques distincts : les clones précoces (en nombre limité) et les clones tardifs. Les corrélations génétiques et les corrélations environnementales ont été calculées pour les 15 caractères. II existe une corrélation génétique positive élevée entre le rendement en matière sèche des tubercules d'une part et le poids frais des tubercules, le poids sec de la plante entière, la durée de vie des feuilles et la durée de la période plantation-floraison d'autre part. Ce dernier caractère apparaît le plus efficace à associer au rendement pour augmenter la réponse à la sélection de celui-ci. Cependant, les possibilités de progrès en utilisant cette relation semblent limitées en raison de la tardivité des floraisons.
\end{abstract}

topinambour / variabilité / héritabilité / corrélation / sélection

Summary - Genetic variability, heritabilities and correlations of 15 characters in a Jerusalem artichoke clone population (Helianthus tuberosus $\mathrm{L}$ ). Fifteen developmental and yield component characters were recorded from 16 different Jerusalem Artichoke clones, sampled among a large clone collection. The clone differences were highly significant, showing a wide range of variation in respect of all characters under study (table I). Broad sense heritability estimates ranged between 19.5 and $96.1 \%$. They were very low for yield related characters and very high for tuber dry matter content and developmental characters. Expected genetic advance with selection of the highest $5 \%$, expressed as the percentage of the mean, varied from 9.5 for emerging time to 30.7 for stalk height (table II). Path analysis was used to partition the genetic correlations between 5 characters into direct and indirect effects. Whole plant dry matter had high positive direct and indirect effects on tuber dry matter yield (tables $I V$ and $V$ ). The mean clone effects for the 15 characters were submitted to a principal component analysis. The first 4 components explained $90 \%$ of the total variation. The dispersion of clones along the first principal component showed the existence of 2 separate morphologic clusters: early clones (a limited number) and late clones (table VI, fig 1). Genetic and environmental correlations between characters were evaluated (table III). Tuber dry matter yield had high positive genetic correlations with fresh tuber weight, whole plant dry matter, green leaf duration and flowering time. Flowering time appeared to be the most efficient character to be associated in a selection for the improvement of tuber yield. However the possibilities for increasing tuber yield by selecting for a later flowering time appeared limited and uncertain.

Jerusalem artichoke / genetic variability / heritability / correlation / selection 


\section{INTRODUCTION}

Le topinambour (Helianthus tuberosus $L$ ) est une composée à multiplication végétative dont les tubercules sont utilisés pour l'alimentation des animaux en hiver. Son importance a considérablement décru au cours des 30 dernières années et il n'était plus cultivé en France, en 1988, que sur 1900 ha.

Cependant plusieurs auteurs ont mis récemment en évidence l'intérêt du topinambour comme source d'inuline, de fructose, d'alcool, de dérivés du furfural et de produits pharmaceutiques divers (Chubey et Dorrell, 1974; Pilnik et Vervelde, 1976; Sachs et al, 1981; Fuchs et Spitters, 1988; Floether, 1989).

Cet intérêt peut être limité par le fait que les clones actuels de topinambour sont sensibles à certaines maladies (oïdium en particulier), ont des tubercules souvent mal conformés et une productivité en glucides faible et variable suivant les années. Sous nos conditions, cette productivité est inférieure de près de $40 \%$ à celle des meilleurs hybrides de betterave à sucre. Nous avons donc un besoin urgent de clones améliorés, plus productifs, mieux adaptés à une intensification et à une mécanisation de la culture.

Le croisement des clones de topinambour est possible à des latitudes proches de celles du centre d'origine de l'espèce $\left(35-40^{\circ} \mathrm{N}\right)$ et les graines issues de ces croisements induisent une nouvelle et importante variabilité (Le Cochec, 1988).

Afin de sélectionner plus efficacement les clones issus de ces graines, il serait utile d'avoir une connaissance effective des liaisons pouvant exister entre le rendement des tubercules et les caractères agronomiques observables sur ces clones. De telles informations ne sont pas disponibles actuellement chez le topinambour.

Le but de cet article a été, à partir d'une population de clones bien différenciés et choisis au préalable, d'estimer, pour différents caractères, la variabilité génétique, l'héritabilité et le gain attendu en sélection. De plus, les corrélations génétiques et les corrélations environnementales, ainsi que la manière dont certains caractères influent sur le rendement en matière sèche des tubercules ont été estimées. L'identification de ces caractères pourrait conduire à la sélection d'un type de plante bien défini.

\section{MATÉRIEL ET MÉTHODES}

Seize clones ont été choisis et multipliés en 1984 à partir d'une collection de 127 clones de topinambour, provenant de 9 pays, et constituée à la Station d'Amélioration des Plantes de Le Rheu au cours des $\mathbf{4 0}$ dernières années.

Les clones choisis sont représentatifs de la variabilité présente dans cette collection pour les caractères étudiés. 7 clones sont originaires de France, 4 clones d'URSS, 3 clones d'Allemagne fédérale, 1 clone de Hongrie et 1 clone est issu de graines récoltées sur des clones précoces.

Ces clones ont été comparés en 1985 dans un essai en blocs randomisés à 5 répétitions. Chaque parcelle comportait 5 lignes de $5 \mathrm{~m}$ de longueur, espacées de $0,70 \mathrm{~m}$. Les plantes, issues d'un seul tubercule, ont été espacées de $0,50 \mathrm{~m}$ sur la ligne. Elles ont été buttées lorsqu'elles atteignaient une hauteur de $0,60 \mathrm{~m}$. La date de plantation a été le 21 avril et la date de récolte des tubercules le 17 décembre. La fertilisation était celle normalement pratiquée dans la région. Aucun traitement fongicide n'a été effectué.

Trois plantes par parcelle ont été choisies au hasard, avant la levée, sur les 3 lignes centrales, à l'exclusion toutefois de la première et de la dernière plante de chaque ligne.

Les 15 observations suivantes ont été faites sur chaque plante :

LEV : nombre de jours entre la plantation et la levée (apparition de la première feuille au-dessus du sol);

$H T J$ : hauteur de la plus haute tige mesurée le 12 juillet (cm);

$F L O$ : nombre de jours entre la plantation et la date de pleine floraison. Par convention ce nombre a été 173 j pour les clones non fleuris, soit $8 \mathrm{j}$ de plus que le clone dont la floraison était la plus tardive;

$O I D$ : note d'intensité d'infection par l'oïdium (Erysiphe cichoraceum) le 20 octobre. Echelle : 0 pas de symptômes; 9 toutes les feuilles couvertes;

DVF : Durée de vie des feuilles : nombre de jours entre la plantation et le noircissement de toutes les feuilles;

$D C V$ : durée du cycle végétatif : nombre de jours entre la plantation et le dessèchement complet de la base des tiges;

$N T I$ : nombre de tiges par plante;

HTR : hauteur de la plus haute tige notée le jour du dessèchement complet des fanes $(\mathrm{cm})$;

NTU : nombre de tubercules de plus de $20 \mathrm{~g}$ par plante;

PSF : poids sec des fanes à la récolte (g). Ces fanes ont été desséchées à l'étuve jusqu'à poids constant; 
$P S E$ : poids sec de la plante entière : fanes et tubercules $(g)$;

$P F T$ : poids frais des tubercules d'un poids supérieur à $20 \mathrm{~g}$ après lavage et séchage à l'air libre;

MST : pourcentage de matière sèche des tubercules : rapport du poids sec au poids frais des tubercules;

PST : poids sec des tubercules (g) déterminé sur des cossettes de $2 \mathrm{~mm}$ d'épaisseur desséchées en étuve à $60^{\circ} \mathrm{C}$ pendant $48 \mathrm{~h}$;

IMU : indice de matière sèche utile : rapport du poids sec des tubercules au poids sec de la plante entière.

Pour chaque caractère, une analyse de la variance a été faite à partir des observations relevées sur chaque plante en utilisant le modèle :

$$
X=M+B l+C l+E r
$$

où $M$ : Moyenne générale, $\mathrm{Bl}$ : effet bloc, $\mathrm{Cl}$ : effet clone, $\mathrm{Er}$ : erreur résiduelle.

Les variances et les coefficients de corrélation génétiques ont ensuite été estimés à partir de la valeur moyenne d'un clone pour chaque caractère.

Le gain génétique attendu en sélection a été déterminé par la formule de Allard (1960) pour un taux de sélection de $5 \%$.

Les coefficients de corrélation environnementaux ont été calculés à partir des coefficients de corrélation phénotypiques et génétiques et de l'héritabilité des 2 caractères, suivant la formule indiquée par Falconer (1960).

Les effets génétiques directs et indirects de 4 caractères sur le rendement en matière sèche des tuber- cules ont été calculés suivant la méthode des moindres carrés décrite par Dewey et Lu (1959).

Enfin, une analyse en composantes principales a été faite à partir de la matrice des corrélations génétiques entre caractères.

Toutes les analyses ont été réalisées en utilisant le logiciel STAT-ITCF.

\section{RÉSULTATS}

\section{Distribution des résidus}

La normalité de la distribution des résidus associés à la population des 16 clones a été testée pour tous les caractères à l'aide des coefficients de Pearson. Les séries observées ne s'écartent pas de façon significative de la normalité à l'exception toutefois du coefficient de symétrie pour 3 caractères (NTI, HTJ et LEV et du coefficient d'aplatissement pour 2 caractères (DDF et $O I D$ ).

\section{Variabilité génétique des caractères}

La moyenne, l'étendue, l'écart type de la moyenne, le coefficient de variation et la valeur du test $F$ des clones sont indiqués pour les 15 caractères sur le tableau I. II apparait, pour tous les caractères, une grande variabilité phénotypique entre clones dans la population considérée.

Tableau I. Variation phénotypique observée pour 15 caractères dans la population de clones.

\begin{tabular}{|c|c|c|c|c|c|}
\hline Caractères & Moyenne & Etendue & $\begin{array}{l}\text { Ecart type de } \\
\text { la moyenne }\end{array}$ & $\begin{array}{l}\text { Cofficient de } \\
\text { variation (\%) }\end{array}$ & $\begin{array}{c}\text { Test } F \text { des } \\
\text { clones }\end{array}$ \\
\hline LEV : Durée plantation-levée (j) & 22,9 & $17,3-29,7$ & 0,9 & 9,0 & $5,6 * *$ \\
\hline HTJ : Hauteur tiges en juillet $(\mathrm{cm})$ & 104,0 & $80,0-132,0$ & 3,1 & 6,6 & $8,3 * *$ \\
\hline FLO : Durée plantation-floraison (j) & 155,1 & $101,0-173,0$ & 0,9 & 1,3 & 347,2 ** \\
\hline OID : Notation oïdium & 7,4 & $2,3-9,2$ & 0,5 & 13,8 & $4,6^{* \star}$ \\
\hline DVF : Durée vie des feuilles (j) & 191,7 & $169,0-215,0$ & 0,6 & 0,7 & $244,4^{\star \star}$ \\
\hline$D C V$ : Durée cycle végétatif (j) & 194,9 & $169,0-217,0$ & 0,9 & 1,0 & $132,7^{\star \star}$ \\
\hline$N T I$ : Nombre de tiges & 2,9 & $1,3-6,0$ & 0,4 & 25,4 & $3,8^{\star \star}$ \\
\hline$H T R$ : Hauteur tiges à la récolte $(\mathrm{cm})$ & 283,5 & $150,0-357,0$ & 8,3 & 6,6 & $34,5 * \star$ \\
\hline NTU : Nombre de tubercules & 38,4 & $16,0-59,3$ & 2,9 & 16,9 & $6,6 * \star$ \\
\hline$P S F$ : Poids sec des fanes $(g)$ & 239,2 & $94,4-347,1$ & 16,3 & 15,3 & 8,8 ** \\
\hline$P S E$ : Poids sec plante entière (g) & 640,1 & $353,4-918,0$ & 40,5 & 14,2 & $3,4^{* *}$ \\
\hline$P F T$ : Poids frais tubercules $(\mathrm{g})$ & 1818,5 & $950,4-2936,4$ & 131,0 & 16,1 & $4,4^{\star \star}$ \\
\hline MST : \% matière sèche des tubercules & 22,2 & $18,3-26,4$ & 0,2 & 2,2 & $72,2^{\star \star}$ \\
\hline PST : Poids sec des tubercules (g) & 399,6 & $237,4-587,8$ & 27,8 & 15,5 & 2,8 ** \\
\hline$I M U$ : Indice de m.s. utile (\%) & 62,9 & $48,4-78,7$ & 1,3 & 4,5 & $22,1^{* *}$ \\
\hline
\end{tabular}

\footnotetext{
** : Significatif au niveau de probabilité $1 \%$
} 
Les coefficients de variation varient de 0,7 à $25,4 \%$ : le plus élevé, de loin, est celui du nombre de tiges et les plus bas, la durée de vie des feuilles, la durée du cycle végétatif, la date de pleine floraison ainsi que le pourcentage de matière sèche de tubercules.

Les coefficients de variation phénotypiques et génétiques ainsi que les variances phénotypiques et génétiques, l'héritabilité et les gains génétiques attendus en sélection (en valeur absolue et en pourcentage de la moyenne) sont indiqués sur le tableau II.

Les coefficients de variation phénotypique sont généralement largement supérieurs aux coefficients de variation génétique. Cependant, ils en diffèrent peu pour la durée du cycle végétatif et de vie des feuilles, la date de floraison, le pourcentage de matière sèche et la hauteur des tiges à la récolte.

Les taux d'héritabilité, au sens large, varient de 19,5 à $96,1 \%$. Ils sont très bas pour les caractères liés au rendement : poids sec et frais des tubercules, poids sec de la plante entière, nombre de tiges ainsi que durée de la levée. Ils sont, par contre, très élevés pour tous les caractères concernant la durée et l'importance du développement de la plante (date de la floraison, durée de vie des feuilles et du cycle végétatif, hauteur des tiges) ainsi que le pourcentage de matière sèche des tubercules.
Les gains génétiques attendus en sélection varient de $9,5 \%$ de la moyenne pour les durées de levée $(2,2$ j) à $30,7 \%$ pour la hauteur des tiges à la récolte $(86,9 \mathrm{~cm})$.

\section{Corrélations entre caractères}

Les corrélations génétiques et les corrélations environnementales entre les différents caractères sont indiquées dans le tableau III.

\section{Corrélations génétiques}

L'examen de ces corrélations permet de constater de très fortes liaisons génétiques entre tous les caractères morphologiques et physiologiques liés à la durée et à la vigueur de la plante : poids des fanes et de la plante entière, hauteur des tiges, durée de vie des feuilles, durée du cycle végétatif et date de floraison. Ce groupe de caractères est positivement relié à la teneur en matière sèche des tubercules c'est-à-dire que les clones tardifs ont généralement des teneurs en matière sèche plus élevées. Ces clones présentent aussi un moindre développement des attaques d'oïdium.

Les caractères de durée et de vigueur sont très négativement liés au nombre de tubercules

Tableau II. Cœfficients de variation et variances phénotypiques et génétiques, héritabilité et gains génétiques attendus en sélection pour les 15 caractères de la population de clones.

\begin{tabular}{|c|c|c|c|c|c|c|c|}
\hline Caractères & $\begin{array}{c}\text { Cofficient } \\
\text { phéno- } \\
\text { typique }\end{array}$ & $\begin{array}{l}\text { de varia } \\
\text { géné- } \\
\text { tique }\end{array}$ & $\begin{array}{c}\text { ation } \\
\text { phéno- } \\
\text { typique }\end{array}$ & $\begin{array}{c}\text { riance } \\
\text { géné- } \\
\text { tique }\end{array}$ & $\begin{array}{l}\text { Héritabilité } \\
\text { (\%) }\end{array}$ & $\begin{array}{l}\text { Gain en } \\
\text { sélection }\end{array}$ & $\begin{array}{l}\text { Gain en } \\
\text { sélection } \\
\text { (en \% de la } \\
\text { moyenne) }\end{array}$ \\
\hline LEV : Durée plantation-levée (j) & 18,6 & 9,2 & 18,15 & 4,48 & 24,7 & 2,20 & 9,5 \\
\hline HTJ : Hauteur tiges en juillet $(\mathrm{cm})$ & 12,3 & 8,2 & 162,8 & 72,2 & 44,4 & 11,71 & 11,2 \\
\hline FLO : Durée plantation-floraison (j) & 11,0 & 10,8 & 291,0 & 279,6 & 96,1 & 33,77 & 21,8 \\
\hline OID : Notation oïdium (0-9) & 23,5 & 13,1 & 3,03 & 0,94 & 30,9 & 1,11 & 15,0 \\
\hline DVF : Durée vie des feuilles (j) & 5,3 & 5,1 & 102,1 & 96,4 & 94,4 & 19,65 & 10,2 \\
\hline DCV : Durée cycle végétatif (j) & 5,0 & 4,9 & 95,9 & 90,5 & 94,4 & 19,04 & 9,8 \\
\hline$N T I$ : Nombre de tiges & 50,9 & 25,5 & 2,18 & 0,55 & 25,0 & 0,76 & 26,2 \\
\hline HTR: Hauteur tiges à la récolte $(\mathrm{cm})$ & 18,8 & 16,7 & 2846,8 & 2252,1 & 79,1 & 86,93 & 30,7 \\
\hline NTU : Nombre de tubercules & 30,9 & 18,4 & 141,2 & 51,1 & 36,2 & 8,86 & 23,1 \\
\hline PSF : Poids sec des fanes (g) & 32,5 & 19,8 & 6041,7 & 2245,1 & 37,2 & 59,63 & 24,9 \\
\hline$P S E$ : Poids sec plante entière $(g)$ & 27,4 & 12,4 & 30726 & 6338 & 20,6 & 74,40 & 11,6 \\
\hline$P F T$ : Poids frais des tubercules $(\mathrm{g})$ & 30,6 & 16,0 & 309107 & 84605 & 27,4 & 313,8 & 17,3 \\
\hline MST : \% matière sèche des tubercules & 8,9 & 8,0 & 3,87 & 3,16 & 81,7 & 3,31 & 14,9 \\
\hline PST : Poids sec des tubercules (g) & 29,6 & 13,1 & 13964 & 2726 & 19,5 & 47,47 & 11,9 \\
\hline MU : Indice de m.s. utile (\%) & 11,8 & 8,6 & 54,64 & 29,57 & 54,1 & 8,19 & 13,1 \\
\hline
\end{tabular}




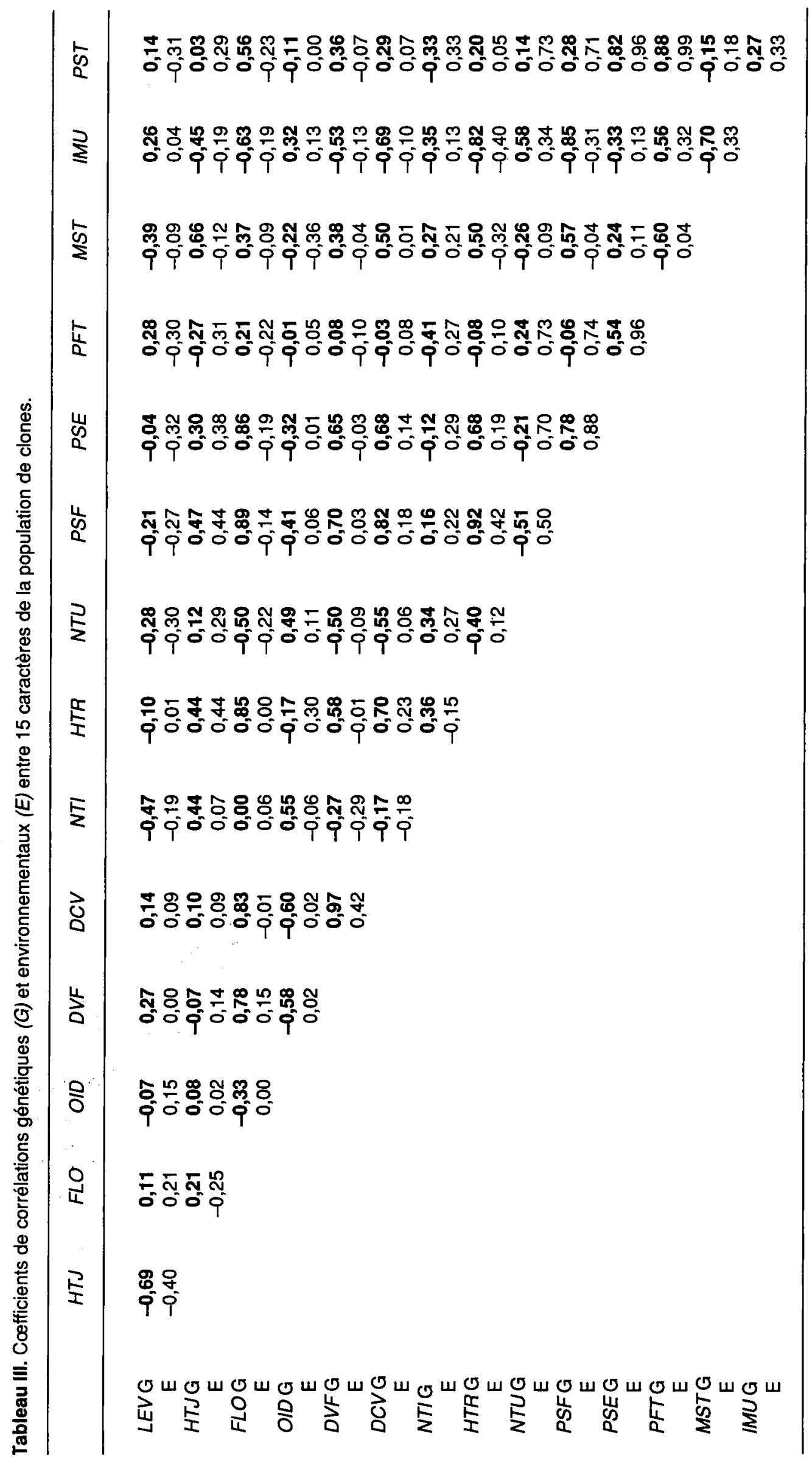


et à l'indice de matière sèche utile. Les clones tardifs ont peu de tubercules et un développement des fanes relativement plus important. Cela se traduit aussi par une forte liaison négative entre la teneur en matière sèche et l'indice de matière sèche utile.

Une longue durée de levée a pour conséquence de réduire la hauteur des tiges en juillet mais ce retard est compensé par la suite.

La forte liaison négative entre le poids frais et la teneur en matière sèche des tubercules était attendue.

Il est intéressant de constater que le poids sec des tubercules, c'est-à-dire le rendement utile, est fortement lié, à la fois au poids frais des tubercules $(0,88)$, au poids sec de la plante entière $(0,82)$ et à la durée plantation-floraison $(0,56)$, ces 2 derniers caractères présentant, comme nous l'avons vu, une forte liaison $(0,86)$. On peut enfin noter que la liaison négative entre le poids $\mathrm{sec}$ et la teneur en matière sèche des tubercules est très faible $(-0,15)$.

\section{Corrélations environnementales}

Elles sont généralement faibles et inférieures aux corrélations génétiques correspondantes. Gallais et al (1983) indiquent cependant que la comparaison de ces 2 types de corrélation est importante à considérer car elle fournit des précisions sur la liaison génétique entre caractères. $\mathrm{Si}$ les 2 corrélations évoluent dans le même sens, cette liaison est due vraisemblablement à des effets pléiotropiques difficiles à rompre par la sélection, alors que si elles évoluent en sens inverse, l'hypothèse d'une liaison entre les 2 caractères est la plus probable. On notera en particulier la présence de pléiotropie pour les caractères de poids frais des tubercules et poids sec de la plante entière. Par contre un effet de linkage peut être soupçonné dans les liaisons du rendement avec la date de floraison et le nombre de tiges. Dans ces derniers cas les corrélations ne sont cependant pas très élevées.

\section{Analyse des path-coefficients}

Le poids sec des tubercules, c'est-à-dire le rendement en produits utilisables (inuline en particulier) est la résultante de plusieurs facteurs agissant indépendamment ou en interaction avec d'autres facteurs. Les effets génétiques directs et indirects de 4 caractères de durée et de vigueur du développement de la plante sur le rendement sont indiqués sur le tableau IV. Ces 4 caractères ont été choisis en raison de leur corrélation génétique élevée avec le rendement.

Le poids frais des tubercules a un effet direct important sur le rendement $(0,54)$ et cet effet direct est notablement renforcé par un effet indirect dû au poids sec de la plante entière.

Le poids sec de la plante entière $a$ un effet direct très élevé sur le rendement $(0,72)$. II intervient également par des effets indirects positifs importants sur les 3 autres caractères. Dans ce sens, il apparaît comme un caractère fondamental, conditionnant une production élevée en matière sèche des tubercules. Une explication physiologique de cette situation sera discutée cidessous.

Tableau IV. Effets génétiques directs et indirects de 4 caractères sur le rendement en matière sèche des tubercules de topinambour. PFT : poids frais des tubercules; PSE : poids sec de la plante entière; FLO : durée plantationfloraison; DVF : durée de vie des feuilles.

\begin{tabular}{ccccccc}
\hline \multirow{2}{*}{ Caractères } & \multicolumn{5}{c}{ Effets indirects dûs à : } & $\begin{array}{c}\text { Corrélation } \\
\text { génétiques } \\
\text { avec PST }\end{array}$ \\
\cline { 2 - 6 } & Effet direct & PFT & PSE & FLO & DVF & 0,88 \\
PFT & 0,54 & - & 0,39 & $-0,06$ & 0,00 & 0,82 \\
$P S E$ & 0,72 & 0,29 & - & $-0,23$ & 0,04 & 0,51 \\
FLO & $-0,27$ & 0,11 & 0,62 & - & 0,05 & 0,36 \\
\hline DVF & 0,06 & 0,04 & 0,47 & $-0,21$ & - & \\
\hline
\end{tabular}


Tableau V. Valeurs moyennes de 4 caractères corrélés avec le poids sec des tubercules (PST) pour 16 clones de topinambour.

\begin{tabular}{lccccc}
\hline Clones & $\begin{array}{c}P S T \\
(g)\end{array}$ & $\begin{array}{c}P F T \\
(g)\end{array}$ & $\begin{array}{c}P S E \\
(g)\end{array}$ & $\begin{array}{c}F L O \\
(j)\end{array}$ & $\begin{array}{c}D V F \\
(j)\end{array}$ \\
\hline & & & & \\
VR & & & 728 & 170 & 194 \\
83 & 479 & 2283 & 695 & 172 & 194 \\
13 & 457 & 2133 & 703 & 165 & 194 \\
NK & 450 & 2083 & 706 & 163 & 195 \\
K8 & 431 & 2021 & 688 & 164 & 193 \\
85 & 423 & 1952 & 641 & 154 & 196 \\
35 & 418 & 1938 & 689 & 159 & 187 \\
F60 & 1692 & 685 & 163 & 194 \\
133 & 417 & 1591 & 635 & 163 & 191 \\
30 & 408 & 1874 & 637 & 156 & 194 \\
120 & 396 & 1666 & 633 & 102 & 214 \\
28 & 388 & 1844 & 627 & 148 & 170 \\
D19 & 387 & 1572 & 506 & 120 & 194 \\
79 & 377 & 2018 & 655 & 149 & 175 \\
17 & 375 & 1643 & 468 & 183,9 & 183 \\
43 & 360 & 1610 & 564 & 2,7 & 191,7 \\
Moyenne & 335 & 1175 & 641,1 & 115,0 & 1,8 \\
ppds 0,05 & 294 & 1818,4 & 370,7 & & \\
\hline
\end{tabular}

L'effet direct des 2 caractères de durée (durée plantation-floraison et durée de vie des feuilles) est respectivement faible et très faible et leur corrélation avec le rendement ne se manifeste que par un effet indirect dû au poids sec de la plante entière.

L'effet résiduel $(0,22)$ est le résultat des erreurs d'échantillonnage et des facteurs non contrôlés intervenant sur l'expression du rendement.

Les valeurs moyennes du poids sec des tubercules et des 4 caractères corrélés sont indiqués pour les 16 clones dans le tableau V. II existe, pour les 5 caractères, des différences très significatives entre clones.

\section{Analyse en composantes principales}

Le tableau VI donne la liste des caractères clés associés à chacun des 4 axes principaux, ainsi que leurs coefficients de corrélation. Ces 4 axes expliquent $90,0 \%$ de la variation totale observée.

Le premier axe est très négativement corrélé avec tous les caractères de durée et de vigueur de la plante qui sont, nous l'avons vu, fortement

Tableau VI. Coefficients de corrélation entre les caractères clés et les composantes principales.

\begin{tabular}{lcccc}
\hline \multicolumn{1}{c}{ Composantes principales } & 1 & 2 & 3 & 4 \\
\hline \multirow{2}{*}{ Caractères clés } & & PST 0,70 & NTU 0,63 & OID 0,55 \\
& PSF - 0,97 & PFT 0,84 & PST 0,61 & \\
& DCV -0,92 & NTI -0,74 & PFT 0,52 & \\
& DVF -0,83 & LEV 0,64 & & \\
& FLO -0.92 & & & \\
& HTR -0,87 & & & \\
& PSE -0,78 & & 15,2 & 7,3 \\
\hline
\end{tabular}


liés. II est positivement corrélé avec l'indice de matière sèche utile.

Le second axe est caractéristique du poids sec et du poids frais des tubercules, ces 2 caractères s'opposant au nombre de tiges par plante.

Le troisième axe semble associer le même caractère, poids sec des tubercules, au nombre de tubercules par plante. Le quatrième axe représente en particulier la différence de comportement des clones vis-à-vis de l'oïdium.

La distribution des clones dans le plan défini par les axes 1 et 2 (fig 1) indique, par rapport à l'axe 1, une nette opposition entre, d'une part, les 2 clones précoces, D19 et 17 et, d'autre part, tous les autres clones qui sont des clones tardifs. Ces 2 clones se caractérisent par une floraison avancée de 35-40 j, des tiges nombreuses et peu développées, un indice de matière sèche utile élevé, une plus grande sensibilité à l'oïdium et des tubercules petits, nombreux, souvent mal conformés et à plus faible teneur en matière sèche. Les clones sont classés par rapport à l'axe 2, en fonction du poids sec et frais des tubercules ainsi que du nombre de tiges par tubercule. A l'extrémité de l'axe les clones VR (Violet de Rennes) et 83 sont caractérisés par un rendement élevé et un nombre de tiges réduit.

\section{DISCUSSION}

Dans la population de clones étudiée, le poids sec des tubercules présente à la fois une faible héritabilité au sens large $(19,5 \%)$ et un faible gain attendu en sélection (11,9\%). La sélection pour le rendement lui-même sera donc difficile et il peut être plus efficace d'agir simultanément sur les facteurs présentant les corrélations génétiques les plus élevées avec le rendement. Nous examinerons successivement ces différents facteurs.

Le poids frais des tubercules est le caractère pour lequel les corrélations génétiques $(0,88)$ et environnementales $(0,99)$ avec le rendement sont les plus élevées. Les caractères poids frais et poids sec des tubercules sont donc très proches et cette liaison n'est que partiellement affectée par la corrélation inverse $(-0,60)$ existant entre le poids frais et le pourcentage de matière sèche des tubercules. Une situation analogue peut être observée dans les variétés de betterave fourragère entre le poids, la teneur en matière sèche et le rendement en matière sèche des racines. En matière de critère de sélection la connaissance du poids frais des racines n'a pourtant pas un très grand intérêt puisqu'il suffit

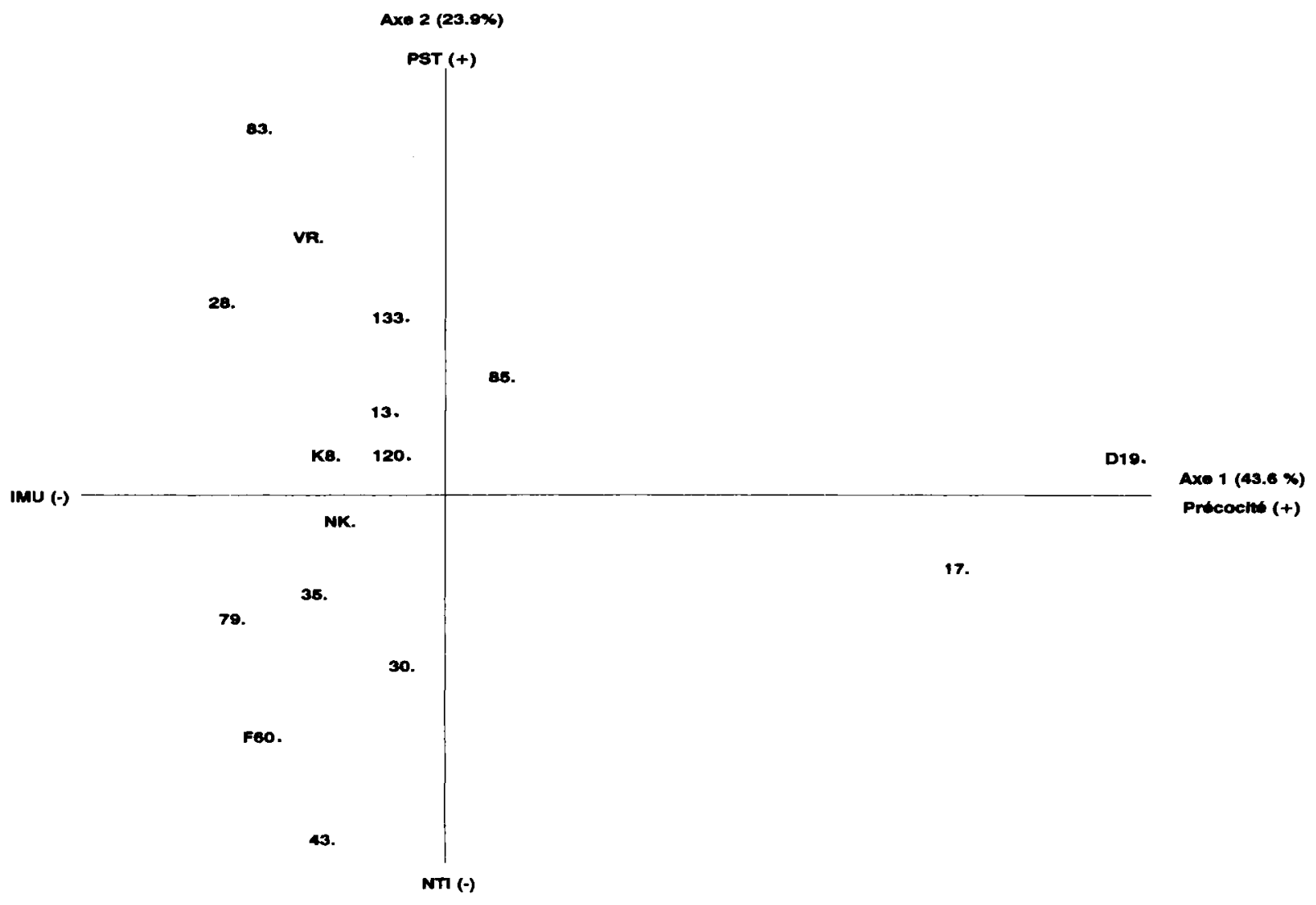

Flg 1. Position relative des clones dans le plan défini par les axes principaux 1 et 2 . 
d'une simple détermination par échantillonnage de la teneur en matière sèche pour avoir une connaissance réelle du rendement.

L'importance du caractère poids sec de la plante entière ressort de l'analyse des pathcoefficients. Il est fortement lié (coefficient de corrélation : 0,82 ) au rendement, du fait de phénomènes de pléiotropie. Le même type de phénomène pourrait expliquer la liaison du rendement au poids sec des fanes (coefficient de corrélation: 0,78 ). L'augmentation du poids sec de la plante entière résulterait donc d'une augmentation parallèle du poids sec des tubercules et des fanes. Chez le topinambour, les tiges sont des organes temporaires de stockage des réserves glucidiques et ces réserves migrent ensuite vers les tubercules en fin de végétation (Incoll et Neales, 1970). La photosynthèse directe ayant une importance réduite, ces réserves caulinaires constituent la source principale des glucides présents dans les tubercules (Barloy, 1989). Un rendement élevé en matière sèche des tubercules résulte d'abord d'une capacité élevée de stockage des glucides dans la moelle des tiges. Cette capacité peut être estimée, assez imparfaitement, par le poids sec des fanes (coefficient de corrélation : 0,28 ), mais elle est, à poids égal, plus élevée dans les clones tardifs, comme VR et 83 ayant un nombre réduit de tiges bien développés, que dans les clones précoces.

La durée plantation-floraison est aussi un caractère possédant une liaison génétique élevée avec le rendement $(0,56)$. Ce caractère est également fortement corrélé $(0,86)$ au poids sec de la plante entière. On peut donc penser que la sélection de variétés à date de floraison aussi tardive ou plus tardive que Violet de Rennes permettrait d'accroître le poids total de la plante et donc le poids sec des tubercules. Gallais et al (1983) indiquent aussi que pour qu'il soit plus efficace de sélectionner sur un caractère lui-même (1) plutôt que sur le caractère lié (2) il faut que l'héritabilité $\left(h_{2}\right)$ du caractère lié soit assez élevée (supérieure à celle $\left(h_{1}\right)$ du caractère sélectionné) et que leur corrélation génétique $r_{1,2}$ soit telle que $r_{12} h_{2}>h_{1}$. Ces 2 conditions sont largement vérifiées pour le caractère durée plantationfloraison. La sélection simultanée pour le rendement et pour ce caractère lié génétiquement au rendement (bien plus héritable que le rendement lui-même) peut être comparée, selon ces auteurs, à un apport de répétitions supplémentaires relativement à un dispositif où seul le rendement serait sélectionné. L'observation de la date de pleine floraison est facile et relativement précise dans le courant de la saison. Elle est cependant moins évidente pour les clones les plus tardifs qui commencent à fleurir durant les premiers jours d'octobre à une époque où la réduction de la photopériode et la baisse des températures interrompent rapidement l'initiation des floraisons.

La durée de vie des feuilles, qui mesure la persistance des feuilles fonctionnelles sous nos conditions, est encore un caractère qui pourrait être associé à la sélection pour le rendement. Cependant, la corrélation génétique entre les 2 caractères $(0,36)$ est assez faible. De plus, l'observation de ce caractère n'est pas toujours possible. En effet, si, en conditions normales, la vie des feuilles des clones tardifs se prolonge jusqu'en fin novembre, les gelées précoces peuvent provoquer, certaines années, le dessèchement prématuré de toutes les feuilles.

En définitive, la sélection pour une longue durée plantation-floraison semble la méthode la plus appropriée pour obtenir des clones dont le poids sec de la plante entière, et donc, le poids sec des tubercules, soit plus élevé que les meilleurs clones actuels. Cependant en raison de la faible possibilité d'intervention sur cette durée, on peut craindre que les progrès dans cette direction demeurent lents et limités. Cette dernière remarque concerne les corrélations obtenues sur un large échantillon de clones. Elle n'est pas nécessairement applicable à la souspopulation des clones précoces dont l'entité a été démontrée par l'analyse des composantes principales.

\section{REMERCIEMENTS}

L'auteur remercie vivement les deux lecteurs de cet article : $M$ Lefort-Buson et $C$ Huygue.

\section{RÉFÉRENCES}

Allard RW (1960) Principles of plant breeding (J Wiley and Sons, eds) New York, London, $484 \mathrm{p}$

Barloy $J(1989)$ Yield elaboration of Jerusalem Artichoke. Topinambour (G Grassi, G Gosse, eds). Comm Eur Communities Eur Rep 11855. DG XII F4. B 1049. Brussels, 65-84

Chubey BB, Dorrell DG (1974) Jerusalem Artichoke, a Potential Fructose Crop for the Prairies. Can Inst Food Sci Technol J 7, 98-100

Dewey DR, Lu KH (1959) A correlation and PathCoefficient Analysis of components of crested Wheatgrass seed production. Agron J 51, 515-518 
Falconer DS (1960) Introduction to quantitative genetics (Oliver and Boyd, eds). Edinburgh and London, $365 \mathrm{p}$

Floether E (1989) Zur Verwertung von Topinambur. Landtechnik 44, 5, 170-171

Fuchs A, Spitters CJT (1988) Jerusalem Artichoke (Helianthus tuberosus L), a potential new crop with non-food applications in the Netherlands. Poster First Int Symp on Fructan, July 26-29, Bonn, 12 p

Gallais A, Vincourt P, Bertholleau JC (1983) Étude de critères de sélection chez le maïs-fourrage : héritabilités, corrélations génétiques et réponse attendue en sélection. Agronomie 3, 751-760
Incoll LD, Neales TF (1970) The stem as a temporary sink before tuberization in Helianthus tuberosus L. $J$ Exp Bot 21, 469-476

Le Cochec $F$ (1988) Jerusalem Artichoke breeding. Topinambour (G Grassi, G Gosse, eds) Comm Eur Commun. EUR Rep 11855. DGXII F4. B 1049 Brussels, 119-124

Pilnik W, Vervelde GJ (1976) Jerusalem Artichoke (Helianthus tuberosus $\mathrm{L}$ ) as a source of Fructose, a Natural Alternative Sweetener. Z Acker Pflanzenb 142, 153-162

Sachs RM, Low CB, Vasavada A (1981) Fuel Alcohol from Jerusalem Artichoke. Calif Agric, Sept-Oct 1981, 4-6 